\title{
Effect of Dietary Pattern and Nutritional Status on Oral Health of Patients Attending the Dental Clinic in University College Hospital, Ibadan, Nigeria
}

\author{
Abimbola Magret Muyide ${ }^{1}$, Motunrayo Taibat Oduneye ${ }^{2}$ \\ ${ }^{1}$ Post Graduate Student, Department of Human Nutrition, University of Ibadan, Nigeria. \\ ${ }^{2}$ Senior Dietician, Dietetics Department, University College Hospital, Ibadan, Nigeria.
}

Corresponding Author: Abimbola M. Muyide

\begin{abstract}
Introduction: Close relationship exists between diet, nutrition and dental health. Oral tissues are nutrition-dependent; inadequate diets can precipitate oral diseases and vice-versa. The study aimed to examine the effect of dietary pattern and nutritional status on the oral health of adults attending the Dental Clinic in University College Hospital, Ibadan.

Methodology: A descriptive cross-sectional study was conducted among 250 participants above 18 years; visiting the dental clinic. Questionnaire was used to obtain information on socio-demographic characteristics, oral habits and oral health-related dietary problems. Oral diagnosis and medical history were obtained from case notes. Validated Food Frequency and 24-Hour Recall questionnaires were used to collect information on frequency and pattern of food and nutrient intakes.

Result: Participants' mean nutrient intakes were adequate for macronutrients but inadequate for nearly all micronutrients. Vitamin $\mathrm{C}$ showed statistical significance with Fracture, Vitamin B6 and B12 with Malocclusion, and Phosphorus and Zinc with Gingivitis. Oral problems affected food intake of $58.4 \%$ of participants, while $52.8 \%$ reportedly changed their diets due to oral problems. Coping mechanisms included chewing on one side of the jaw (40.8\%), opting for liquid/soft foods $(11.2 \%)$, avoiding hard foods $(25.6 \%)$, omitting meat, fruits and vegetables from meals $(21.6 \%)$.

Conclusion: The study establishes the prevalence of hidden hunger in patients with dental problem and the close relationship between nutrition and oral health. Nutrition education on the importance of adequate diet for good oral health is required, and treatment of oral health problems should involve Registered Dietitian Nutritionists.
\end{abstract}

Keywords: Dietary pattern, Nutritional status, Oral health, Coping mechanisms, Nigeria

\section{INTRODUCTION}

Nutrition is defined as the intake of food, considered in relation to the body's dietary needs, while diet refers to the sum of what we eat and drink. Oral health is defined as having healthy teeth as well as "being free of chronic oral-facial pain conditions, oral and pharyngeal (throat) cancers, oral soft tissue lesions, birth defects such as cleft lip and palate, and scores of other diseases and disorders that affect the oral, dental, and craniofacial tissues, collectively known as the craniofacial complex', [1

Nutrition and diet have a local effect on the oral cavity. Oral tissues like all tissues in the body are nutrition-dependent. Nutrition affects the teeth during development and malnutrition may exacerbate periodontal and oral infectious diseases. ${ }^{[2]}$ Also important is the manner in which nutrition affect the health of the oral tissues, the quantity and quality of saliva and general health of the body. So also, oral health determines the type of food 
consumed, taste perception and ultimately the nutritional level. Although a risk factor for micronutrient deficiency, oral diseases are often hidden and invisible, or they are accepted as an unavoidable consequence of life and ageing. The high burden of oral diseases globally represents a widely underestimated public health challenge for almost all countries worldwide. ${ }^{[3,4]}$

The key nutrition problem involving the oral cavity is the inability to maintain adequate oral food intake. Studies have shown that it is likely that poor nutrition and diet can affect the development and integrity of the teeth leading to rapid progression of dental diseases. Therefore, there may exists a vicious cycle where poor oral health affects the nutritional status, which in turns leads to defects in development and maturation of teeth structure or protective mechanism, predisposing teeth to dental diseases. ${ }^{[1,5]}$

The Global Burden of Disease Study in 2017 estimated that oral diseases affect 3.5 billion people worldwide and a vast majority of adults have dental cavities worldwide, often leading to pain and discomfort. ${ }^{[6]}$ In Nigeria, studies done by the Federal Ministry of Health on oral health in 2012 shows that an accurate assessment of the oral health situation in Nigeria is difficult because of the absence of coordinated system for collecting oral health data in the country. Unfortunately, this is still the present reality in Nigeria. However, the limited information available suggests that the oral health of Nigerians is poor and the need for oral health care among Nigerians is huge. ${ }^{[7]}$ Studies indicate that periodontal disease occurs in Nigerians at an early age with a prevalence of $15-58 \%$ in persons over 15 years. Traumatic injuries are common in the oro-facial region and there is an apparent rise in the occurrence of oral tumors and dental caries among Nigerians. ${ }^{[7,8]}$ Appropriate medical, dental and nutritional interventions are needed to improve dietary intake and nutritional status of people to prevent them from becoming malnourished.
Adequate nutrition and good health are the right of all individuals and also form a basis for the development of a nation. There is a public health concern about oral diseases, and available evidence shows there is lack of awareness of the populace about the importance of oral health, misconception that oral diseases are neither debilitating nor life-threatening and poor understanding of the fact that good nutrition promotes oral health as oral health also promotes good nutrition and overall well-being of people. This therefore is necessitating an increase in ways in which dieticians and health professional can help prevent poor oral health associated with malnutrition and poor dietary patterns and vice versa.

Studies on the effect of nutritional status on oral health are sparse in Nigeria and there is a continuous concern on how oral health affects the overall health of the whole populace and the future of Nigeria. Knowledge of changes in nutritional status due to poor oral health will not only help in better management of nutritional problems, but will also enable better clinical outcome. The purpose of this study therefore was to examine the effect of dietary pattern and nutritional status on the oral health of adults. The study further aimed to determine the relationship between dietary pattern, nutritional status and oral health of adult patients attending the dental clinic in University College Hospital, Ibadan.

\section{METHOD}

A descriptive cross-sectional study was conducted among adults (18 years and above), attending the Dental clinic (Oral and Maxillofacial Unit) of the University College Hospital, Ibadan, Nigeria. The participants were selected using purposive sampling technique. It was estimated that a sample size of 374 was required to achieve a level of precision with a standard error of $5 \%$ using a $95 \%$ confidence interval (CI) and a prevalence of dental caries of $58 \%$ for the calculation. [8] An interviewer administered questionnaire was used to obtain information on socio-demographic 
characteristics (which included age, gender, marital status, employment status and educational level), oral habits and oral health related dietary problems. Anthropometric measures were collected; oral diagnosis and medical history were obtained from case notes as participants were been seen by the dentist. Validated Food Frequency and 24-Hour Recall questionnaires were also used to collect information on frequency and pattern of food and nutrient intakes.

The study proposal was reviewed and approved by the University of Ibadan/University College Hospital Ethics Committee (UI/UCH EC), UCH Ibadan. Informed consent was obtained from the participants. Participation in the study was voluntary and no incentive was offered.

Data was analyzed using Statistical Package for Social Sciences (SPSS version 21) and TDA (Version 1.0.001) software. Descriptive statistics such as frequency, percentages, mean, and standard deviation was used to present the results. Chi-square test was used to investigate if the association between oral health and BMI of the respondents was statistically significant. Regression analysis was used to determine the effect of dietary pattern and nutritional status on the oral health of respondents. The independent variables (IV) are the dietary pattern and nutritional status while the dependent variable (DV) is the oral health. A $p$-value of $\leq 0.05$ was accepted as statistically significant.

\section{RESULT}

\section{Socio-demographic characteristics:}

Majority of the participants were females (63.2\%). Most participants were found to be single $(63.2 \%)$ with tertiary level of education $(76.8 \%)$.

Information on the anthropometric measurement of the participants is shown in Tables 1 below.

Table 1: Anthropometry of Participants

\begin{tabular}{|l|l|l|l|}
\hline Anthropometry & Frequency(N) & Min-Max & Mean \pm S.D \\
\hline Age & 250 & $18.0-66.0$ & $33.1 \pm 14.5$ \\
\hline Weight $(\mathrm{kg})$ & 250 & $45.0-115.0$ & $65.6 \pm 13.3$ \\
\hline Height $(\mathrm{cm})$ & 250 & $150-196$ & $170 \pm 10$ \\
\hline BMI $(\mathrm{kg} / \mathrm{m} 2)$ & 250 & $17.0-42.2$ & $23.6 \pm 4.2$ \\
\hline
\end{tabular}

Oral health status: Toothache $(40.8 \%)$ and mal-alignment $(24.8 \%)$ were the most common reasons why people visited the dental clinic. And at the time of study, most respondents had oral health problems of painful chewing $(40.8 \%)$ and sensitive teeth $(36.0 \%)$. Also, periodontal disease $(32.2 \%)$, malocclusion $(24.7 \%)$ and dental caries (17.8\%) were the most occurring diagnosed oral disease among those treated at the clinic.

Association between oral health problems and BMI of participants is shown in Table 2 below:

Table 2: Association between Oral health problems and BMI of Respondents

\begin{tabular}{|l|l|l|}
\hline & BMI & $\boldsymbol{P}$-value \\
\hline Oral Health Problem & Red of swollen gum & 0.019 \\
& Painful chewing & 0.013 \\
& Sensitive teeth & 0.005 \\
& Bad breath & 0.392 \\
& Tender or bleeding gum & 0.754 \\
& Loose tooth & 0.094 \\
\hline \multirow{2}{*}{$*$ Chi-square test } & $*$-value statistically significant at $p \leq 0.05$
\end{tabular}

The Chi-square test done to show the association between the diagnosed oral health diseases and BMI of respondents showed a statistical significance of 0.001 (at $p \leq 0.05$ )

Oral health and food intake: $58.4 \%$ of participants reported that oral health problem affected their food intake while $52.8 \%$ reported that they had changed their diets due to oral health problems. Majority of the respondents whose food intake had being affected by oral health problems developed a coping mechanism by opting for chewing food with one side of the jaw or avoiding some foods $(40.8 \%)$ while some opted for liquid/soft foods (11.2\%); most participants further had to stop eating hard foods like nuts and roasted corn (25.6\%), meat/ bone (14.4\%) and fruits/vegetables (7.2\%). Nearly all participants had no other 
health challenge $(89.6 \%)$ while $9.6 \%$ had other health challenges like hypertension.

Hidden hunger present in most participants is shown in Table 3 below; containing information on the mean nutrient intakes of the participants and the adequacies. The average amounts of energy and macronutrients, minerals and vitamins consumed using a 24-Hour Recall are shown.

Table 3: Nutrient Intake and Adequacy of Respondents

\begin{tabular}{|l|l|l|l|l|}
\hline Tutrients & N & Min - Max & Mean \pm S.D & RDA \\
\hline Calories (kcal) & 250 & $582.9-4289.5$ & $1900.7 \pm 553.3$ & Inadequate \\
\hline Protein (g) & 250 & $13.0-126.7$ & $64.8 \pm 22.6$ & Adequate \\
\hline CHO (g) & 250 & $98.3-736.1$ & $313.5 \pm 90.9$ & Adequate \\
\hline Fat (g) & 250 & $4.6-125.7$ & $46.7 \pm 20.9$ & Adequate \\
\hline VitA (mcg) & 250 & $32.5-1276.8$ & $749.8 \pm 266.0$ & Adequate \\
\hline VitC (mg) & 250 & $0.6-91.8$ & $16.6 \pm 20.0$ & Inadequate \\
\hline Vit B2 (mg) & 250 & $0.04-2.0$ & $0.8 \pm 0.5$ & Inadequate \\
\hline Vit B6 (mg) & 250 & $0.0-1.7$ & $0.6 \pm 0.4$ & Inadequate \\
\hline Vit B12 (mcg) & 250 & $0.0-100.0$ & $1.8 \pm 6.3$ & Inadequate \\
\hline Calcium (mg) & 250 & $0.9-1268.1$ & $329.7 \pm 252.7$ & Inadequate \\
\hline Phosphorus (mg) & 250 & $0.0-1181.8$ & $506.2 \pm 271.9$ & Inadequate \\
\hline Potassium (mg) & 250 & $7.8-4978.9$ & $1012.0 \pm 867.7$ & Inadequate \\
\hline Zinc (mg) & 250 & $0.0-21.8$ & $11.4 \pm 4.2$ & Adequate \\
\hline Iron (mg) & 250 & $0.7-25.0$ & $15.8 \pm 4.6$ & Adequate \\
\hline
\end{tabular}

Please note: Nutrient intake of the respondents is compared with FAO/WHO 2002 standard for Recommended Daily Allowance (RDA) of nutrients.

Using regression analysis to determine the relationship between nutritional status (adequacy) and diagnosed oral health, Carbohydrate and Vitamin C showed statistical significance with Fracture, Vitamin B6 and B12 showed statistical significance with Malocclusion and Phosphorus and Zinc showed statistical significance with Gingivitis. A $p$-value of $\leq$ 0.05 was accepted as statistically significant.

\section{DISCUSSION}

According to Papas et al. life-style characteristics have been found to affect food intake and dietary quality adversely and these include low educational attainment, marital status and socioeconomic status. [9] Dependent individual's food intake and dietary quality can also be affected by those they depend on. Some of the common reasons suggested to affect food choices in different individuals include ignorance, availability, cost and financial resources. Therefore, it is necessary to determine the background characteristics of participants to know the socio-demographic components that effect their dietary pattern and nutritional status in relations to oral health. In this study, the socio-demographic characteristics used include sex, age, marital status, educational level and employment status.

There was significant relationship between carbohydrate and gender, fat and educational level and phosphorus and educational level. The participants who have acquired (or are acquiring) some form of higher education and are believed to also have some knowledge on the importance of healthy eating and might make healthier food choices (for dental health), however Contento et al. states that knowledge alone does not enable people to adopt healthier eating behaviors. ${ }^{[10]}$ Financial status which is usually determined by employment status can also influence food choices in the individuals. Over half $(56.8 \%)$, of the participants were employed while $43.2 \%$ were either unemployed or retired meaning that their financial status might be affecting their food choices and hence can affect their nutritional and oral health status. In addition, $63.2 \%$ of the participants were single whiles $36.8 \%$ were married. According to Story et al. eating behaviors are also strongly influenced by social environments, which include school, family, friends, and peer networks. ${ }^{[11]}$ 
Energy is required in the body for metabolic processes, physiological functions, muscular activity, heat production, growth, and the synthesis of new tissues and protein. Mean energy intake for the participants was $1900.7 \mathrm{kcal}$ which was slightly below recommended level but met the percent energy contributions requirement for protein, carbohydrate and fat. This may be due to under-estimation of food and nutrients during data collection that might have reflected in nutrient analysis since participants may not be able to recall exact amounts of foods eaten.

When compared to their RDAs, participants' mean nutrient intakes were found to be inadequate for most of the vitamins and minerals except vitamin $\mathrm{A}$, zinc and iron. Essential nutrient like calcium that is necessary for healthy teeth was found inadequate. Vitamin $\mathrm{C}$ which has been discovered to play a role in maintaining and repairing healthy connective tissue along with its antioxidant properties was also found inadequate. Potassium, whose deficiency can cause a sensation of a raw, sore tongue and burning mouth when eating, drinking, or talking (it can also cause gingival bleeding) was also deficient, amongst many others. This is similar to studies by Sheetal et al. and Rugg-Gunn who also reported that micronutrient deficiency affects the integrity of the oral cavity. ${ }^{[12,13]}$

From this study, periodontal disease, malocclusion and dental caries were the most occurring oral disease among those treated at the clinic. This is comparable to global reports and other studies on oral health burdens. ${ }^{[14,15]}$ Also, oral problems affected food intake of over half of the participants in this study and this resulted in change or modification of diets. Changes and modifications made to food will definitely affect one's enjoyment of his/her favorite food, limit food choices and sometimes result in total avoidance of some food items. The omission of food items like meat, nuts, fruits and vegetables (which most participants omitted) from diets may lead to the exclusion of vital micronutrients, leading to hidden hunger. This also, is similar to previous reports that stated that poor dentition and oral health results in the avoidance of food which calls for thorough chewing and people with tooth loss and gum disease are likely to limit their diets to softer foods. ${ }^{[16]}$ In addition, the natural source of fiber, fruits and vegetables which are also excellent sources of vitamins and minerals, are excluded and variety is limited hence nutrient deficiency follows. ${ }^{[16]}$ Likewise, under-nutrition worsens the severity of oral infections and may eventually lead to their development into life-threatening diseases. ${ }^{[17,18]}$ People who have lost some teeth can be affected by their dentition and this can lead to reduced intakes of fruit and vegetables, some foods that require proper mastication and some key nutrients. ${ }^{[19]}$

The limitation of the study was that the time frame for the study was relatively short and multiple strikes by doctors and all federal health workers which shut down the clinic affected the study. Hence, of the original sample size of 374 respondents, only 250 participated in the study.

\section{CONCLUSION}

The study establishes the prevalence of hidden hunger in patients with dental problem and the close relationship between nutrition and oral health. Malnutrition resulted in dental diseases and also, poor oral health resulted in sub-optimal and or unhealthy eating habits which further worsen both oral health and malnutrition. In addition, the harmful effects of this vicious cycle of poor oral health on nutritional status and vice versa can induce metabolic syndrome such as obesity and hypertension and also reduce quality of life.

The most appropriate intervention recommended is that of an effective dietary counseling in dental clinics (especially to patients with recurring oral problems and those with eating difficulties.). Dietary changes will not only improve the oral health (and vice versa), but most importantly improve the general health. It is 
time that registered dietitian nutritionists to be part of the dental health team. Also, similar studies should be conducted on more specific population groups.

\section{Acknowledgement: None}

\section{Conflict of Interest: None}

\section{Source of Funding: None}

\section{Ethical Approval: Approved}

\section{REFERENCES}

1. World Health Organization. World Oral Health Report. Continuous improvement of oral health in the 21st century - The approach of the WHO Global Oral Health Programme. Geneva: World Health Organization; 2003. 38p. Report No.:WHO/NMH/NPH/ORH/03.2

2. Ghosh A, Pallavi SK, Nagpal B, et. al. Nutrition and Oral Health: A Review. Indian Journal of Applied Research; 2015; 5(11): 546-549.

3. FDI World Dental Federation. The Challenge of Oral Disease - A call for global action. The Oral Health Atlas (2nd ed.). Geneva: FDI; 2015.120p. ISBN: 978-2-9700934-8-0

4. Leena Smadi \& OmayahNassar. Oral \& Dental Health Knowledge and Attitude among Nursing Students. World Journal of Educational Research; 2016;3(2): 238-250.

5. Kagihara LE, Niederhauser VP, Stark M. Assessment, management, and prevention of early childhood caries. J Am Acad Nurse Pract. 2009 Jan;21(1): $1-10$.

6. Kassebaum NJ, Smith AGC, Bernabé E, et. al. Oral Health Collaborators.Global, regional, and national prevalence, incidence, and disability-adjusted life years for oral conditions for 195 countries, 1990-2015: a systematic analysis for the global burden of diseases, injuries, and risk factors. 2017 J Dent Res. 96(4):380-387.
7. Akpata, E.S. Oral Health in Nigeria. International DentalJournal. 2004; 54. 361-6.

8. Adegbembo AO, El-Nadeef MA, Adeyinka A. National survey of dental caries status and treatment needs in Nigeria. Int Dent J. 1995 Feb;45(1):3544.

9. Papas MA, Alberg AJ, Ewing R, et. al. The built environment and obesity. Epidemiol Rev. 2007;29:129-43.

10. Content IR, Manning AD \& Shannon B. Research perspective on school-based nutrition education. J Nutr Edu 1992 (24): 247-260.

11. Story M, Neumark-Sztainer D, French S. Individual and environmental influences on adolescent eating behaviors. J Am Diet Assoc. 2002 Mar;102(3 Suppl):S40-51.

12. Sheetal A, Hiremath VK, Patil AG, et. al. Malnutrition and its oral outcome - a review. J Clin Diagn Res. 2013;7(1):178-180.

13. Rugg-Gunn AJ. Nutrition, diet and oral health. Journal of the Royal College of Surgeons of Edinburgh. $2001 \mathrm{Dec} ; 46(6)$ : 320-328.

14. Benjamin RM. Oral health: the silent epidemic. Public Health Rep. 2010; 125(2):158-159.

15. Bratthall D, Petersen PE, Stjernswärd JR, et al. Oral and Craniofacial Diseases and Disorders. In: Jamison DT, Breman JG, Measham AR, et al., editors. Disease Control Priorities in Developing Countries. 2nd edition. Washington (DC): The International Bank for Reconstruction and Development / The World Bank; 2006. Chapter 38.

16. Burton B., and Foster W. R. (1988). Human Nutrition, 4th Edition. McGraw Hill Book Company. Pg. 248-252

17. Syafiqah, Izzatul \& Nuraeny, Nanan \& Herawati, Erna. (2016). Nutritional status of vitamin B12 on small group of dental students based on food frequency questionnaire. Padjadjaran Journal of Dentistry. 28. 
Abimbola Magret Muyide et.al. Effect of dietary pattern and nutritional status on oral health of patients attending the dental clinic in University College Hospital, Ibadan, Nigeria.

18. Vernetti-Callahan, D. Eating Well For A Healthy Mouth. Journal for Continuiting Education Course. 2013. Pg 1-21.

19. Walls AW, Steele JG. The relationship between oral health and nutrition in older people. Mech Ageing Dev. 2004 Dec;125(12):853-7.
How to cite this article: Muyide AM, Oduneye MT. Effect of dietary pattern and nutritional status on oral health of patients attending the dental clinic in University College Hospital, Ibadan, Nigeria. Int $J$ Health Sci Res. 2021; 11(6): 374-380. DOI: https://doi.org/10.52403/ijhsr.20210656 